\title{
Convergence Analysis of Transcendental Fractals
}

\author{
Shafali Agarwal \\ Research Scholar, Singhania University, \\ Rajasthan, India
}

\author{
Ashish Negi \\ Phd, Dept. of Computer Science, G.B. Pant \\ Engg. College, Pauri Garwal, Uttarakhand, India
}

\begin{abstract}
Mandelbrot set with transcendental function presents an out of the ordinary field of research because of its magnificence and geometrical complexities. Earlier many researchers used feedback system to study it and reveal new concepts unexplored the geometry of transcendental Mandelbrot set with new iterative approach. The objective of the paper is to analyze the work done by several researchers to visualize beautiful fractal graphics of Mandelbar set with sine, cosine functions using Mann iteration method. Also the rate of convergence of function in the presence of transcendental function and conjugacy with Mandelbrot set and related complex structure of superior Julia set.
\end{abstract}

\section{General Terms}

Computer Graphics, Fractal

\section{Keywords}

Transcendental superior Julia set, AntiJulia set, Fixed Point, Mann Iteration, Escape Time Fractal.

\section{INTRODUCTION}

French mathematician Gaston Julia in 1918 investigated the iteration process of complex function and attained a Julia set and gave a direction to the fractal world [4]. Later Benoit Mandelbrot in 1979 studied a very complex \& perturbed structure which is known as Mandelbrot set. A lot of researches carried out to unveil the geometrical and functional ambiguity of both the sets [14]. We are analyzing fractal images that have been generated from iterating polynomials of

type $\bar{z}^{d}+c$ with transcendental function defined in the complex plan. Here $c$ is a complex parameter, $d$ is an integer, bar sign denotes complex conjugation \& transcendental function can be sine, cosine or tangent. It has been observed that after applying much iteration to these polynomials, complex number $z$ either move away from the origin and escape towards infinity or bounded to origin. Points with bounded orbit under iteration make up the filled in Julia set, which we call $K_{c}^{d}$ for the holomorphic and $K_{c}^{* d}$ for the antiholomorphic case. The topological boundaries of these bounded sets are Julia sets [3]. Points bounded to origin converge to a fixed point. Fixed points can be classified in three categories:

Asymptotically stable: Means all nearby solutions converge to it.

Stable: Means all nearby solutions stay nearby.

Unstable: Means mostly all nearby solutions diverge away from the fixed point.

Authors discussed about the singular values that plays an important role in determining the dynamics of complex system. According to them any attracting fixed point must have a singular value in its immediate basin of attraction [15]. A Transcendental entire function possesses critical and asymptotic values. Here a critical value of function is such as there exists a $z_{0}$ belongs to $c$ and $f\left(z_{0}\right)=c 1 \& f^{\prime}\left(z_{0}\right)=0$. In this case, $z_{0}$ is called a critical point of $f$ and an asymptotic value of $f$ is such as there exists a curve "any symbol" tending to infinity and $f(z)->a$ as $z->\infty$ along "any symbol" [5]. The fractal image of superior Mandelbrot set consists of small mini Mandelbrot set like images found in the scattered surrounding of it is known as the midgets [1]. The study of midgets in the Mandelbrot set is given by Philip [13] and Romera [16]

Fractal with conjugate of $z$ referred to as Mandelbar set, name is given by Crowe et al. because of its formal analogy with Mandelbrot set and also brought its features bifurcations along axes rather than at points. The critical point of Mandelbar set is zero as in Mandelbrot set [20]. The study of connectedness locus for antiholomorphic polynomials defined as Tricorn, coined by Milnor, plays intermediate role between quadratic and cubic polynomials [6]. Recently Shizuo [11] has quoted the Multicorn as the generalized Tricorn or the Tricorn of higher order and presented beautiful images and their various properties. This set is particularly interesting because its topological properties are different from the ones believed to be true for the Mandelbrot set.

Shafali et al. have applied the concept of conjugacy to transcendental Mandelbrot set and generated fractals [see 17, $18,19]$. They have iterated the function to compute a precise value for the fixed point and hence the efficiency of function depends on the rate of convergence of the polynomial. Our paper focuses on the relative analysis of all those functions and to ascertain that which function converges faster to fixed point than other. We will also see the beautiful Julia set images of those functions.

\section{PRELIMINARIES \& DEFINITIONS}

The formation of fractal images is achieved by using feedback system. There are basically two types of feedback machines i.e. one-step machine and two-step machine. Both types of machines can be characterized by iterative procedures. Iteration methods are one way to achieve the self-similarity exhibited by fractals [2]. One-step feedback machines are characterized by Peano-Picard iterations represented by the formula $x_{n+1}=f\left(x_{n}\right)$ where $f\left(x_{n}\right)$ can be any function. It is very useful mathematical tool and has been developed in particular for the numerical solution of complex problems. In two-step feedback machine, the output is computed by the formula $x_{n+1}=f\left(x_{n}, x_{n-1}\right)$, which requires two numbers as input and returns a new number as output[12]. Other related definitions which will be required for subsequent analysis:

(Superior iterates) Let $X$ be a non-empty set of real numbers and $f: X->X$. For $x_{0}$ belongs to $X$, construct a sequence $\left\{x_{n}\right\}$ in the following manner $[8,9]$ :

$x_{1}=\beta_{1} f\left(x_{0}\right)+\left(1-\beta_{1}\right) x_{0}$

$x_{2}=\beta_{2} f\left(x_{1}\right)+\left(1-\beta_{2}\right) x_{1}$

$x_{n}=\beta_{n} f\left(x_{n^{-1}}\right)+\left(1-\beta_{n}\right) x_{n^{-1}}$

where $0<\beta_{n}<=1$ and $\left\{\beta_{n}\right\}$ is convergent away from 0 . 
The sequence $\left\{x_{n}\right\}$ constructed this way is called a superior sequence of iterates, denoted by $S O\left(f, x_{0}, \beta_{n}\right)$ At $\beta_{n}=1, S O\left(f, x_{0}\right.$, $\beta_{n}$ ) reduces to $O\left(f, x_{0}\right)$. This procedure was essentially given by Mann, first to study it for $\beta_{n}$ in 1955 [21]. Since the results obtained in fractal modelling via Mann iterates are the super set of their corresponding fractal models in the Picard orbit.

(Superior Orbit) The sequence $x_{n}$ constructed above is called Mann sequence of iteration or superior sequence of iterates. We denote it by $S O\left(x_{0}, s, t\right)$.

Escape-time fractals (or superior orbit fractals)

Many Julia and Mandelbrot sets have been computed for polynomials in superior orbits. In the literature these are called superior Julia sets and superior Mandelbrot sets respectively and are defined by Rani and Kumar [8, 9] as follows:

(Superior Julia sets) The set of complex points SK whose orbits are bounded under superior iteration of a function $Q$ is called the filled superior Julia set. A superior Julia set SJ of $Q$ is the boundary of the filled superior Julia set [9].

(Superior Mandelbrot sets) A Superior Mandelbrot set SM for a function of the form $Q_{c}(z)=z_{n}+c, n=1,2, \ldots$, is defined as the collection of $c \in C$ for which the superior orbit of the point 0 is bounded,

$\mathrm{SM}=\left\{c \in C:\left\{Q_{c}^{k}(0): k=0,1, \ldots ..\right\}\right.$ is bounded in $\left.\mathrm{SO}\right\}$ [8].

To compute SJ and SM sets, Rani and Kumar [10] gave new and considerably improved escape criterions and called them superior escape criterions. For a complex map of the form $Q_{c}^{k}=z^{n}+c$, the escape criterion is $\max \left\{|c|,(2 / s)^{1 / n-1}\right\}$ and for the cubic polynomial $Q_{a, b}(z)=z^{3}+a z+b$, the escape criterion is $\max \left\{|b|, \quad(|a|+2 / s)^{1 / 2}\right\}$. Similarly for the quadratic polynomial $Q_{a, b}(z)=z^{2}+c$, the escape criteria is $\max \{|c|$, $2 / s\}$.

Superior Tricorn and Multicorn: A superior multicorn, for the complex-valued polynomial $A c=\bar{z}^{d}+c$, for $d \geq 2$, is the collection of all $c \varepsilon C$, for which superior orbit $S O\left(A_{c}, z_{0}, \mathrm{~s}\right)$, with $z_{0}=0$ is bounded, i.e.

$A c=\left\{c \& C:\left\{A_{c}^{k}(0), d=0,1,2, \ldots\right\}\right.$ is bounded with respect to $S O\}$.

\begin{tabular}{|l|l|l|l|}
\hline $\begin{array}{c}\text { Number } \\
\text { of } \\
\text { Iteration i }\end{array}$ & \multicolumn{1}{|c|}{$\mathbf{F ( z )}$} & $\begin{array}{c}\text { Number } \\
\text { of } \\
\text { Iteration i }\end{array}$ & $\mathbf{F}(\mathbf{z})$ \\
\hline 11 & 1.1485 & 16 & 1.1589 \\
\hline 12 & 1.1546 & 17 & 1.1590 \\
\hline 13 & 1.1572 & 18 & 1.1591 \\
\hline 14 & 1.1583 & 19 & 1.1591 \\
\hline 15 & 1.1588 & 20 & 1.1591 \\
\hline
\end{tabular}

TABLE 1: Orbit of $F(z)->\sin \left(z^{2}\right)+c$ at $s=0.3 \&$ (C=0.75-0.03125i)

Here we skipped 10 iteration and the value converges to a fixed point after 17 iterations
A superior multicorn with $d=2$ is known as superior tricorn [7].

Suppose $x_{0}$ is a fixed point for $F$. Then $x_{0}$ is an attracting fixed point if $\left|F^{\prime}\left(x_{0}\right)\right|<1$. The point $x_{0}$ is a repelling fixed point if $\left|F^{\prime}\left(x_{0}\right)\right|>1$. Finally if $\left|F^{\prime}\left(x_{0}\right)\right|=1$, the fixed point is neutral [14].

Note that the initial value $z_{0}$ should be infinity, since infinity is the critical point of $z$ for transcendental polynomial. However instead of starting with $z_{0}=\infty$, it is simpler to start with $z_{1}=c$, which yields the same result. A critical point of $z$ $\rightarrow f(z)+c$ is a point where $f^{\prime}(z)=0$. The point $z$ in Mandelbrot set for transcendental function has an orbit that satisfies $\operatorname{imag}(z)>50$, then the orbit of $z$ escapes[14].

\section{FIXED POINT CALCULATION USING MATLAB AND RESULT DISCUSSION}

The process of fractal generation uses iterative method on Mandelbrot set i.e. $f(z)=z^{n}->c$, where $z$ and $c$ both are complex quantities and $n>1$. We are considering fractal generation process for transcendental Mandelbar set using escape time method. The fractal images are constructed as a twodimensional array of pixels. Each pixel is represented by a pair of $(x, y)$ co-ordinates. If the fractal image is constructed with various values of $z$ keeping the value of $c$ constant is known as $z$ plane images on the other hand if fractal image is constructed for various values of $c$ keeping the value of $z$ constant is known as $c$ plane images. Here we are analyzing the work done by various researchers in the fractal world.

In the Dynamics of Mandelbrot Set with Transcendental Function Shafali, Gunjan \& Ashish studied the fractal images of transcendental polynomial $z->\sin \left(z^{n}\right)+c$ in $z$ plane and $c$ plane using Mann iteration. The main attraction of this paper was the existence of unique images like earthen lamp (diya), Lord Vishnu icon etc by the iteration of quadratic, cubic and bi-quadratic function. Here we have seen that the visual characteristics of images are changed as the value of constant $s$ varies with polynomial.

The following tables show the number of iterations needed to converge the image to fixed point.

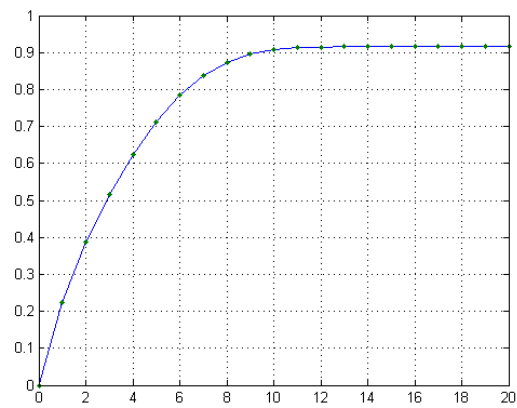

Fig 1: Orbit of $F(z)$ at $s=0.3$ for $(C=0.75-0.03125 i)$ 


\begin{tabular}{|l|l|l|l|}
\hline $\begin{array}{c}\text { Number } \\
\text { of } \\
\text { Iteration i }\end{array}$ & \multicolumn{1}{|c|}{$\mathbf{F}(\mathbf{z})$} & $\begin{array}{c}\text { Number } \\
\text { of } \\
\text { Iteration i }\end{array}$ & $\mathbf{F}(\mathbf{z})$ \\
\hline 1 & 0.1406 & 6 & 0.2282 \\
\hline 2 & 0.2011 & 7 & 0.2283 \\
\hline 3 & 0.2208 & 8 & 0.2283 \\
\hline 4 & 0.2264 & 9 & 0.2283 \\
\hline 5 & 0.2278 & 10 & 0.2283 \\
\hline
\end{tabular}

TABLE 2: Orbit of $F(z)->\sin \left(z^{2}\right)+c$ at $s=0.5 \&$ $(\mathrm{C}=-\mathbf{0 . 2 8 1 2 5 - 0 . 0 4 6 8 7 5 i})$

The value converges to a fixed point after 6 iterations

\begin{tabular}{|l|l|l|l|}
\hline $\begin{array}{c}\text { Number } \\
\text { of } \\
\text { Iteration i }\end{array}$ & \multicolumn{1}{|c|}{$\mathbf{F ( z )}$} & $\begin{array}{c}\text { Number } \\
\text { of } \\
\text { Iteration } \mathbf{i}\end{array}$ & $\mathbf{F}(\mathbf{z})$ \\
\hline 1 & 0.1563 & 6 & 0.1914 \\
\hline 2 & 0.1801 & 7 & 0.1915 \\
\hline 3 & 0.1877 & 8 & 0.1915 \\
\hline 4 & 0.1903 & 9 & 0.1915 \\
\hline 5 & 0.1911 & 10 & 0.1915 \\
\hline
\end{tabular}

TABLE 3: Orbit of $F(z)->\sin \left(z^{2}\right)+c$ at $s=1 \&$ $(\mathrm{C}=0.15625+0.0234375 i)$

The value converges to a fixed point after 6 iterations

In "Fixed Point Results of Transcendental Superior Antifractals" Shafali and Ashish analyzed transcendental polynomial with conjugacy using two step feedback processes. The obtained images are having the features of multicorn with transcendental function. They have used Mann iteration to produce fractal images of function $z->\sin \left(\bar{z}^{d}+c\right)$ and transformation of the function for quadratic, cubic and biquadratic polynomial. All of the fractals possess two common

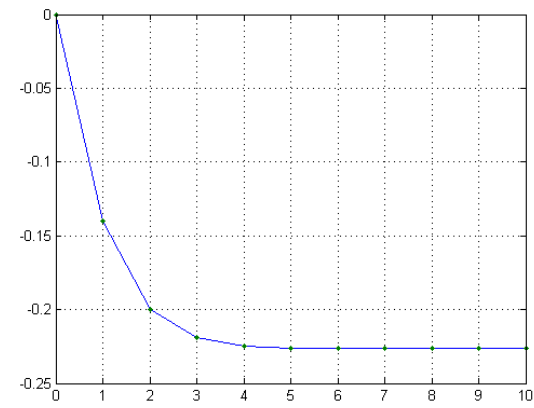

Fig 2: Orbit of $F(z)$ at $s=0.5$ for $(C=-0.28125-0.046875 i)$

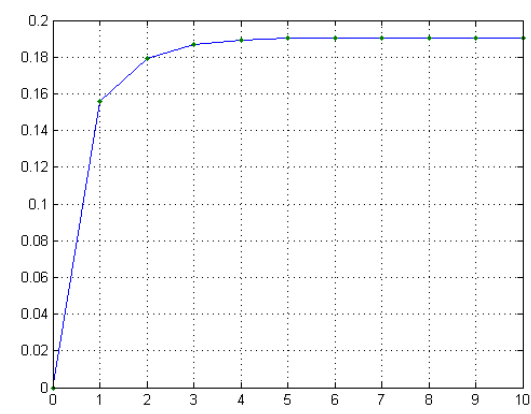

Fig 3: Orbit of $F(z)$ at $s=1$ for $(C=0.15625+0.0234375 i)$

properties for apiece polynomial. One is all of the images generated using function $\sin \left(\bar{z}^{d}+c\right)$ is having number of arms $(d+1)$ for power $d$ and an added is for even power of $d$, images are symmetrical about real axis whereas for odd power of $d$, images are symmetrical about both real and imaginary axis. The tabular representation of fixed point calculation is given below.

\begin{tabular}{|l|l|l|l|}
\hline $\begin{array}{c}\text { Number } \\
\text { of } \\
\text { Iteration i }\end{array}$ & \multicolumn{1}{|c|}{$\mathbf{F}(\mathbf{z})$} & $\begin{array}{c}\text { Number } \\
\text { of } \\
\text { Iteration } \mathbf{i}\end{array}$ & $\mathbf{F}(\mathbf{z})$ \\
\hline 24 & 0.4239 & 30 & 0.4245 \\
\hline 25 & 0.4241 & 31 & 0.4246 \\
\hline 26 & 0.4243 & 32 & 0.4246 \\
\hline 27 & 0.4244 & 33 & 0.4246 \\
\hline 28 & 0.4244 & 34 & 0.4246 \\
\hline 29 & 0.4245 & 35 & 0.4246 \\
\hline
\end{tabular}

TABLE 4: Orbit of $F(z)->\sin \left(\bar{z}^{2}+c\right)$ at $s=0.1 \&$ $(\mathrm{C}=-\mathbf{0 . 7 1 4 2 8 5 7 1 4 3 + 0 . 6 2 5 i})$

Here we skipped 23 iterations and the value converges to a fixed point after 30 iterations

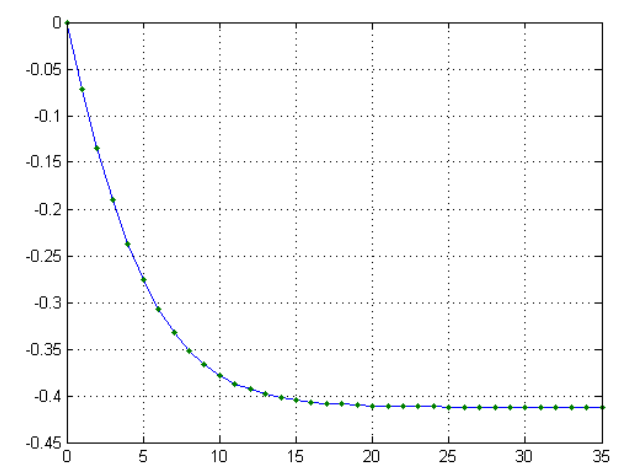

Fig 4: Orbit of $F(z)$ at $s=0.1$ for $(C=-$ $0.7142857143+0.625 i)$ 


\begin{tabular}{|l|c|c|c|}
\hline $\begin{array}{c}\text { Number } \\
\text { of } \\
\text { Iteration } \mathbf{i}\end{array}$ & $\mathbf{F}(\mathbf{z})$ & $\begin{array}{c}\text { Number } \\
\text { of } \\
\text { Iteration } \mathbf{i}\end{array}$ & $\mathbf{F}(\mathbf{z})$ \\
\hline 1 & 0.8375 & 7 & 0.8748 \\
\hline 2 & 0.8864 & 8 & 0.8749 \\
\hline 3 & 0.8712 & 9 & 0.8748 \\
\hline 4 & 0.8760 & 10 & 0.8748 \\
\hline 5 & 0.8745 & 11 & 0.8748 \\
\hline 6 & 0.8750 & 12 & 0.8748 \\
\hline
\end{tabular}

TABLE 5: Orbit of $F(z)->\sin \left(\bar{z}^{2}+c\right)$ at $s=0.5 \&$ $(C=-1.675-0.0375 i)$

The value converges to a fixed point after 8 iterations

\begin{tabular}{|l|l|l|l|}
\hline $\begin{array}{c}\text { Number } \\
\text { of } \\
\text { Iteration i }\end{array}$ & \multicolumn{1}{|c|}{$\mathbf{F}(\mathbf{z})$} & $\begin{array}{c}\text { Number } \\
\text { of } \\
\text { Iteration i }\end{array}$ & $\mathbf{F}(\mathbf{z})$ \\
\hline 1 & 0.0156 & 6 & 0.0155 \\
\hline 2 & 0.0154 & 7 & 0.0155 \\
\hline 3 & 0.0155 & 8 & 0.0155 \\
\hline 4 & 0.0155 & 9 & 0.0155 \\
\hline 5 & 0.0155 & 10 & 0.0155 \\
\hline
\end{tabular}

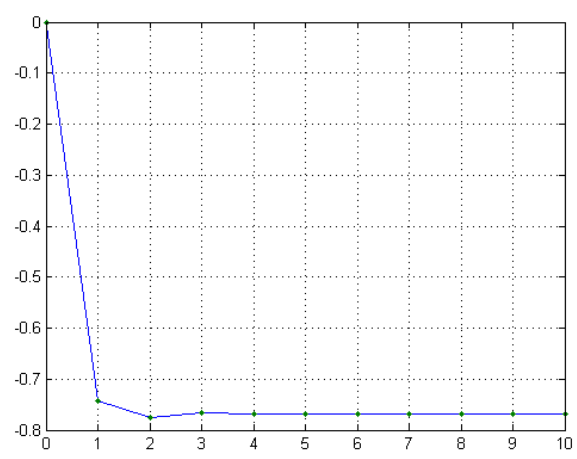

Fig 5: Orbit of $F(z)$ at $s=0.5$ for $(C=-1.675-0.0375 i)$

TABLE 6: Orbit of $F(z)->\sin \left(\bar{z}^{2}+c\right)$ at $s=1 \&$ $(\mathrm{C}=-\mathbf{0 . 0 1 5 6 2 5 - 0 . 0 0 7 8 1 2 5 i )}$

The value converges to a fixed point after 2 iterations

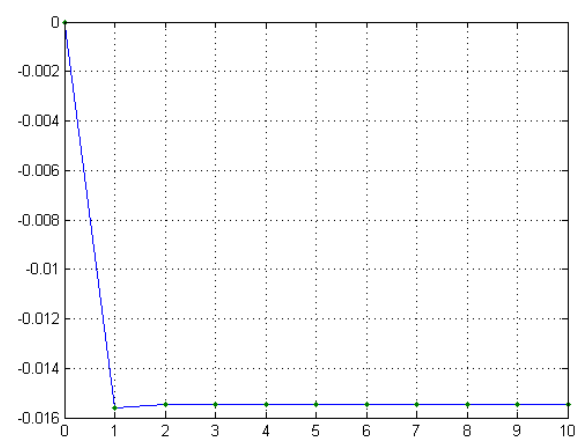

Fig 6: Orbit of $F(z)$ at $s=1$ for $(C=-0.015625$ 0.0078125i)

In "Midgets of Transcendental Superior Mandelbar Set" Shafali and Ashish explored the presence of midgets in Mandelbar set for cosine function using Mann iteration i.e. $z->\cos \left(\bar{z}^{d}+c\right)$. The geometrical shape resembles to both multicorn as well as transcendental polynomial function. Another interesting feature is the existence of midgets in terms of tricorn, multicorn and Mandelbrot sets with different number of bulbs on external rays of key image. It is observed that all the midgets are either basic Mandelbrot set or tricorn irrespective of the degree of polynomial for constant $0<s<1$. However the midgets are dependant on the degree of polynomial at $s=1$.

\begin{tabular}{|l|l|l|l|}
\hline $\begin{array}{c}\text { Number } \\
\text { of } \\
\text { Iteration } \mathbf{i}\end{array}$ & \multicolumn{1}{|c|}{$\mathbf{F}(\mathbf{z})$} & $\begin{array}{c}\text { Number } \\
\text { of } \\
\text { Iteration } \mathbf{i}\end{array}$ & $\mathbf{F}(\mathbf{z})$ \\
\hline 11 & 2.5149 & 16 & 2.5179 \\
\hline 12 & 2.5163 & 17 & 2.5180 \\
\hline 13 & 2.5171 & 18 & 2.5180 \\
\hline 14 & 2.5175 & 19 & 2.5181 \\
\hline 15 & 2.5178 & 20 & 2.5181 \\
\hline
\end{tabular}

TABLE 7: Orbit of $F(z)->\cos \left(z^{2}+c\right)$ at $s=0.1 \&$ $(\mathrm{C}=-\mathbf{1 8 . 8 7 5 + 0 . 2 5 i})$

Here we skipped 10 iterations and the value converges to a fixed point after 18 iterations

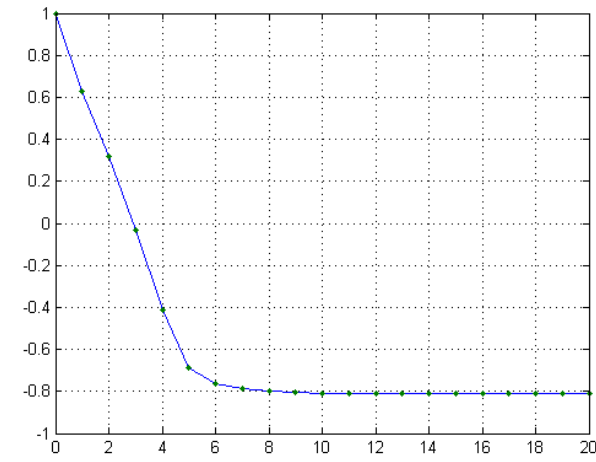

Fig 7: Orbit of $F(z)$ at $s=0.1$ for $(C=-18.875+0.25 i)$ 


\begin{tabular}{|l|l|l|l|}
\hline $\begin{array}{c}\text { Number } \\
\text { of } \\
\text { Iteration i }\end{array}$ & \multicolumn{1}{|c|}{$\mathbf{F ( z )}$} & $\begin{array}{c}\text { Number } \\
\text { of } \\
\text { Iteration i }\end{array}$ & $\mathbf{F}(\mathbf{z})$ \\
\hline 1 & 0.5833 & 6 & 0.5047 \\
\hline 2 & 0.4723 & 7 & 0.5055 \\
\hline 3 & 0.5167 & 8 & 0.5052 \\
\hline 4 & 0.5009 & 9 & 0.5053 \\
\hline 5 & 0.5069 & 10 & 0.5053 \\
\hline
\end{tabular}

TABLE 8: Orbit of $F(z)->\cos \left(z^{2}+c\right)$ at $s=0.5 \&$ $(\mathrm{C}=-\mathbf{0 . 8 3 3 3 3}+0.0416667 \mathrm{i})$

The value converges to a fixed point after 8 iterations

\begin{tabular}{|l|l|l|l|}
\hline $\begin{array}{c}\text { Number } \\
\text { of } \\
\text { Iteration i }\end{array}$ & \multicolumn{1}{|c|}{$\mathbf{F}(\mathbf{z})$} & $\begin{array}{c}\text { Number } \\
\text { of } \\
\text { Iteration i }\end{array}$ & $\mathbf{F}(\mathbf{z})$ \\
\hline 1 & 0.5062 & 6 & 0.4827 \\
\hline 2 & 0.4760 & 7 & 0.4828 \\
\hline 3 & 0.4840 & 8 & 0.4828 \\
\hline 4 & 0.4824 & 9 & 0.4828 \\
\hline 5 & 0.4828 & 10 & 0.4828 \\
\hline
\end{tabular}

TABLE 9: Orbit of $F(z)->\cos \left(Z^{2}+c\right)$ at $s=1 \&$ $(\mathrm{C}=-\mathbf{0 . 4 9 3 7 5 - 0 . 0 5 6 2 5 i})$

The value converges to a fixed point after 6 iterations

\section{GENERATION OF SUPERIOR JULIA} SETS

Transcendental function with superior Julia or AntiJulia set follows the law of having $2 d$ wings, where $d$ is the power of $z$. Most of the images for all polynomials possesses symmetry about both $x$ and $y$ axis. The fractal for $z->\cos \left(\bar{z}^{d}+c\right)$ formed with large central body having rotational and reflection

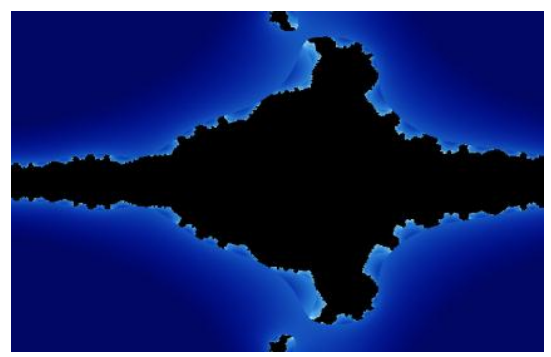

Fig 10: $F(z)=\sin \left(z^{2}\right)+c, s=0.5$ and $c=-\mathbf{0 . 5 8 7 5},-\mathbf{- 0 . 1 2 5 i}$

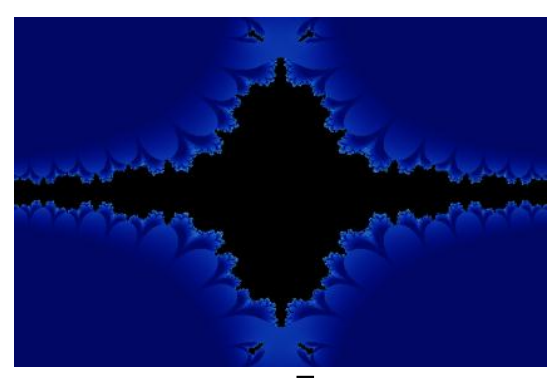

Fig 12: $\mathbf{F}(\mathrm{z})=\cos \left(\bar{z}^{2}+c\right), \mathrm{s}=0.5$ and $c=-0.83333+0.0416667 i$

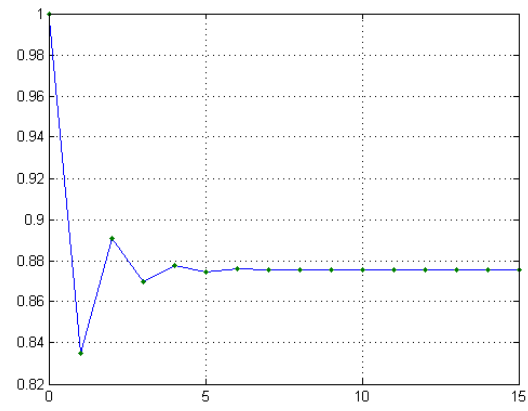

Fig 8: Orbit of $F(z)$ at $s=0.5$ for $(C=-$ $0.83333+0.0416667 i)$

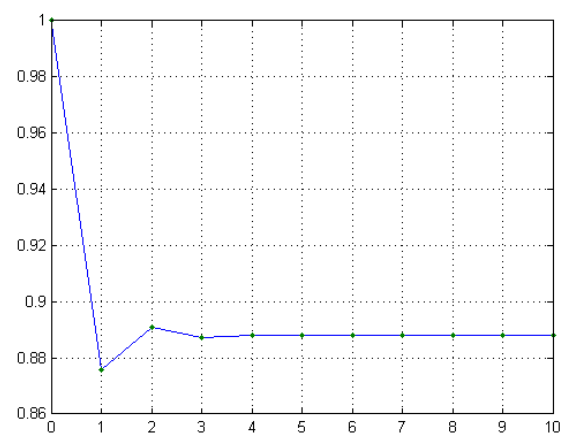

Fig 9: Orbit of $F(z)$ at $s=1$ for $(C=-0.49375-0.05625 i)$

symmetry along with axes symmetry. Here enormously stunning images of transcendental superior Julia and AntiJulia set have been generated for different power of polynomial. The complement of the superior antiJulia set is displayed in blue colour. It appears that antiJulia set consists of uncountably many curves or hairs extending to infinity in the plane.

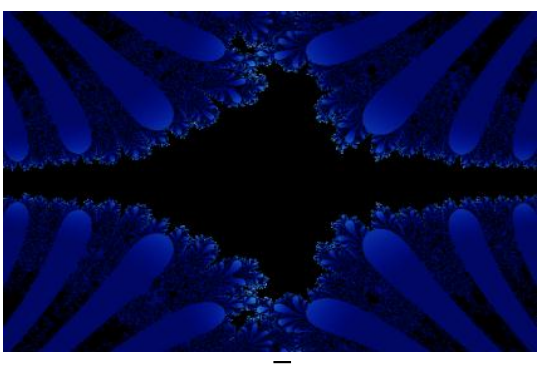

Fig 11: $\mathbf{F}(\mathbf{z})=\sin \left(\bar{z}^{2}+c\right), \mathbf{s}=0.5$ and $c=-0.16667,0.0208333 i$ 


\section{CONCLUSION}

In this paper, we have reviewed the research work done by Ashish, Shafali and Gunjan in the field of fractal. The study have shown the striking properties and escape criteria for transcendental function and generated the corresponding fractals using Mann iteration in which most of the images are having symmetry along $x$ axis and $y$ axis. The analysis represents the beauty of midgets in the form of Mandelbrot set and multicorn appeared in superior antiMandelbrot set for the function $\cos \left(\bar{z}^{d}+c\right)$. In their study we observed fixed points

\section{REFERENCES}

[1] Ashish Negi \& Mamta Rani,"Midgets of Superior Mandelbrot set", Chaos, Solitons and Fractals 36 (2008) 237-245.

[2] C. Pickover, "Computers, Pattern, Chaos, and Beauty", St. Martin"s Press, NewYork, 1990.

[3] Eike, Lau and Dierk Schleicher, "Symmetries of fractals revisited", The Mathematical Intelligencer, Vol 18, No. 1, 45-51, DOI: 10.1007/BF03024816.

[4] G. Julia, "Sur 1' iteration des functions rationnelles", J Math Pure Appli. 8 (1918), 737-747

[5] Kin-Keung Poon, "Fatuo-Julia Theory on Transcendental Semigroups", Bull Austrad. Math Soc. Vol. 58 (1998) [403-410].

[6] J. Milnor, "Dynamics in one complex variable; Introductory lectures", Vieweg (1999).

[7] M Rani,,"Superior Antifractals", volume 1, IEEE, 978-14244-5586-7/10/2010.

[8] M Rani and V Kumar. Superior Mandelbrot sets, J. Korea Soc. Math. Educ. Ser. D; Res. Math. Educ. (8)(4)(2004), 279-291. (44)

[9] M Rani, V. Kumar. Superior Julia set. J Korea Soc Math Educ Ser D Res Math Educ 2004;8(4):261-77. (43)

[10] M. Rani. Iterative procedures in fractals and chaos, $\mathrm{PhD}$ Thesis, Department of Computer Science, Gurukula Kangri Vishwavidyalaya, Hardwar, India, 2002.

[11] N. Shizuo and Dierk Schleicher, "On multicorns and unicorns: I. Antiholomorphic dynamics, Hyperbolic components and real cubic polynomials", Internat. J. Bifurcation. Chaos Applied Science Engineering, (13)(10)(2003), 2825- 2844. of function for different values of constant $s$. An observable fact is that the number of iterations required to achieve convergence is dependant to the value of constant $s$. As the value of $s$ raise to 1 , the image convergence is faster to the fixed point and the fractals converged to asymptotically stable fixed points. Fractal iterates provides envision for fixed points to a given function, allowing us to determine properties with out computing it. In future researcher can apply Mann iteration to study the speed of convergence of new Fractal Models using different function polynomial

[12] Peitgen H, Jürgens H, Saupe D. Chaos and fractals: new frontiers of science. New York: Springer-Verlag; 2004.

[13] Philip AGD. Wrapped midgets in the Mandelbrot set. Computer Graphics 1994;18(2):239-48.

[14] R. L. Devaney, A First Course in Chaotic Dynamical Systems: Theory and Experiment, Addison-Wesley, 1992. MR1202237 Zbl 0768.58001

[15] R. L. Devaney, "Complex Exponential Dynamics", Boston University, August 6, 2000. $\{5\}$

[16] Romera M, Pastor G, Montoya F. On the cusp and the tip of a midget in the Mandelbrot set antenna. Phys Lett A 1996; 221(3-4): 158-62. MR1409563 (97d:58073).

[17] Shafali Agarwal, Gunjan Srivastava, Ashish Negi, "Dynamics of Mandelbrot Set with Transcendental Function". International Journal of Advanced Computer Science \& Applications", Vol. 3 No. 52012 (142-146).

[18] Shafali Agarwal, Ashish Negi, "Fixed Point Results of Transcendental Superior Antifractals". Proceedings of International Conference of Advances in computing, communications and informatics". (In Press)

[19] Shafali Agarwal, Ashish Negi, "Midgets of Transcendental Superior Mandelbar Set". International Journal of Computer Science Issues, Impact Factor 0.242 (In Press)

[20] W. D. Crowe, R. Hasson, P. J. Rippon, and P. E. D. Strain-Clark, "On the structure of the Mandelbar set". Nonlinearity (2)(4)(1989), 541-553. MR1020441 Zbl 0701.58028 .

[21] WR. Mann, "Mean value methods in iteration". Proc Am Math Soc 1953;4:506-10. 